\section{The Need for United States-Based Guidelines for Myeloproliferative Neoplasms}

\author{
Brady L. Stein, MD, MHS; Susan O'Brien, MD; Peter Greenberg, MD; and \\ Ruben A. Mesa, MD
}

In last month's issue of JNCCN-Journal of the National Comprehensive Cancer Network, we, with the help of a members of several NCCN Panels, reviewed rapidly evolving diagnostics, new tools to assess disease burden and prognosis, and expanding treatment options for patients with myeloproliferative neoplasms (MPNs). ${ }^{1}$ Historically, guidance on MPN diagnostic and therapeutic considerations has come from expert consensus opinion, and currently, only societies outside the United States, such as the British Committee for Standards in Haematology, International Working Group for MPN Research and Treatment (IWG-MRT), and European LeukemiaNET (ELN), provide guidance on MPN diagnosis, treatment, and response assessment. ${ }^{2-8}$ We believe that because of changing diagnostic algorithms and, importantly, new therapies, US physicians would benefit from comprehensive United States-based guidelines for the management of MPN.

Physicians already have such guidelines to aid the evaluation and management of other chronic myeloid neoplasms, including myelodysplastic syndromes (MDS) and chronic myelogenous leukemia (CML). ${ }^{9,10}$ We believe several areas of interest within MPNs would align nicely with the goals of NCCN Guidelines, and we are happy to note that NCCN Clinical Practice Guidelines in Oncology (NCCN Guidelines) for MPNs are currently in the planning stages.

We expect these formal guidelines for hematologists and hematologistoncologists will serve a variety of functions regarding diagnosis. First, such guidelines can increase awareness of the currently recognized MPN molecular markers (JAK2 ${ }^{\mathrm{V} 617 \mathrm{~F}}, \mathrm{CALR}$, and MPL) and suggest appropriate clinical settings for molecular testing and monitoring. Second, these guidelines can enable navigation through the diagnostic challenges presented by phenotypic mimicry between MPN subtypes and, occasionally, nonmalignant conditions. As an example, these guidelines could display a differential diagnosis for secondary thrombocytosis, erythrocytosis, and fibrosis, along with diagnostic algorithms that aid in distinction of essential thrombocythemia (ET) from prefibrotic myelofibrosis (MF), polycythemia vera (PV) from JAK2 $2^{\mathrm{V} 617 \mathrm{~F}}$, positive ET, and recognition of post-ET/post-PV MF.

As we noted in the previously published article, modifying thrombosis risk is paramount in the management of ET and PV. ${ }^{1}$ Formal guidelines for hematologists and hematologist-oncologists could provide a consensus-based vascular risk assessment and suggest appropriate clinical indications for and goals of cytoreductive therapies. Hydroxyurea is commonly used, but the evidence base in PV is scarce. We believe that NCCN Guidelines will also help providers weigh efficacy and safety of hydroxyurea, interferon-alpha, and anagrelide and busulfan, given the short- and long-term adverse effects that can occur with each agent.

Duration and choice of anticoagulant therapy is not well established, but consensus is required, along with indications for plateletpheresis. Finally, hematologists and hematologist-oncologists need guidance on how to best prepare patients with MPNs for elective surgery and pregnancy.

Although MPN treatment is in part guided by vascular risk assessment in ET/PV and predicted survival in MF, awareness of the MPN symptom burden is increasing, and accordingly, focus on symptom-directed therapy, which served as a basis for approval of JAK inhibitors, is also increasing. An MPN guideline can emphasize the need for

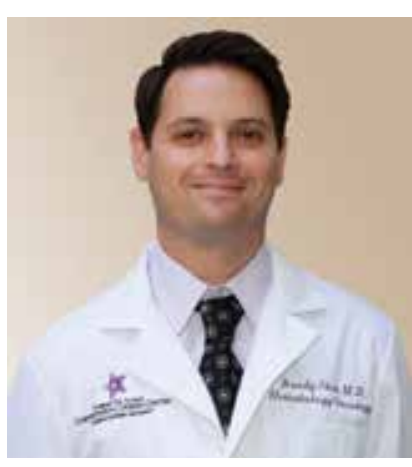

Brady L. Stein, MD, MHS

Brady L. Stein, MD, MHS, received Internal Medicine training and fellowship training in hematology from the Johns Hopkins University School of Medicine. During fellowship, he was an awardee of the K12 Blood Scholars Program, focusing on clinical research in hematology. During that program, he received an MHS in Clinical Investigwation at the Johns Hopkins Bloomberg School of Public Health. He moved to Northwestern University Feinberg School of Medicine in the summer of 2010 as an Attending Physician in Hematology/Oncology.

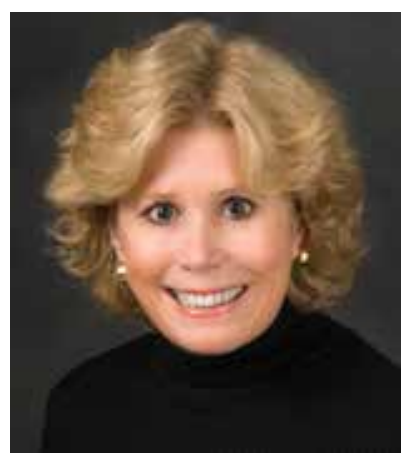

Susan O'Brien, MD

Susan O'Brien, MD, is Associate Director for Clinical Science for the Chao Family Comprehensive Cancer Center and Medical Director of the Sue and Ralph Stern Center for Cancer Clinical Trials and Research at UC Irvine Health, Irvine, California and former Chair of the NCCN Guidelines for chronic myelogenous leukemia.

The ideas and viewpoints expressed in this commentary are those of the author and do not necessarily represent any policy, position, or program of NCCN. 


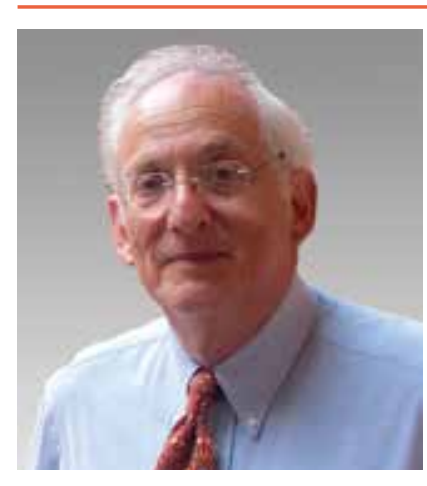

Peter Greenberg, MD

Peter Greenberg, MD, is Professor of Medicine, Hematology Division, at Stanford Cancer Institute, and Chair of the NCCN Guidelines Panel for Myelodysplastic Syndromes.

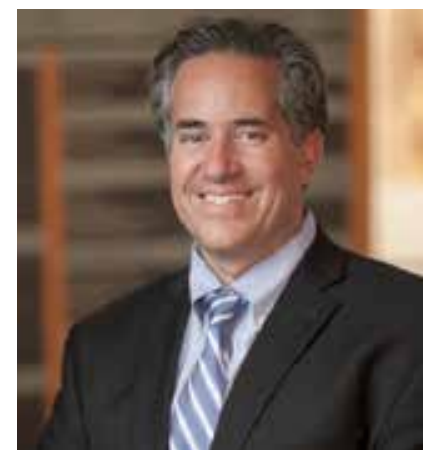

Ruben A. Mesa, MD

Ruben A. Mesa, MD, is Professor of Medicine of Mayo Medical School and chair of the Division of Hematology/Oncology in the Department of Internal Medicine at Mayo Clinic and deputy director at Mayo Clinic Cancer Center in Arizona. Dr. Mesa is active in the Arizona Cancer Coalition. Dr. Mesa attended University of Illinois at Urbana-Champaign for his undergraduate degree and earned his medical degree at Mayo Medical School. He completed both a residency in internal medicine and a fellowship in hematology/ medical oncology at Mayo Graduate School of Medicine. symptom assessment when planning therapy, and outline a practical supportive care approach.

Further, ruxolitinib is approved for MF, and may be approved for use in patients with $\mathrm{PV}$ in the near future, and 2 additional JAK inhibitors (pacritinib and momelotonib) are in phase III development. We believe that NCCN Guidelines for MPN will help identify appropriate candidates for JAK-inhibitor therapy and outline appropriate dose modifications in response to safety or efficacy concerns, and tapering in the event of discontinuation due to the potential for rebound symptoms. Drug-refractory splenomegaly is sometimes encountered, even in the JAK-inhibitor era; therefore, guidelines that aid in appropriate patient selection for splenectomy would aid in decision-making. Additionally, guidance on when to incorporate radiation therapy into the management of splenic or nonhepatosplenic extramedullary hematopoiesis is needed.

Stem cell transplantation is the only curative therapy for MF, and providers need advice on how to appropriately select candidates for this high-risk but high-reward procedure. Finally, an unprecedented number of clinical trials have been performed evaluating novel therapeutics as single agents or in combination. As data mature, physicians will need guidance on how to incorporate such strategies into clinical practice.

Last month's review focused primarily on ET, PV, and MF, but hematologists often encounter other MPNs that present diagnostic and therapeutic challenges and also lack guideline representation. One example includes systemic mastocytosis (SM), in which molecular evaluation for the KIT ${ }^{\mathrm{D} 816 \mathrm{~V}}$ mutation-present in more than $80 \%$ of patients; laboratory evaluation of serum tryptase levels; and immunohistochemical staining of mast cell aggregates (eg, tryptase, CD117, CD25) in bone marrow or other extracutaneous organs are key to establishing WHO minor and major criteria for the disease. ${ }^{11}$ Although imatinib is not appropriate for patients with $\mathrm{KIT}^{\mathrm{D} 816 \mathrm{~V}}$ mutations, the tyrosine kinase inhibitors midostaurin and masitinib are under evaluation.

Patients with SM also have unique treatment needs that include not only avoidance of triggers but also reduction in the burden of mediator-release symptoms, bone destruction, and anaphylaxis. Chronic eosinophilic leukemias and hypereosinophilic syndromes can have distinct clinical consequences, including cardiovascular, neurologic, pulmonary, dermatologic, and gastrointestinal complications, and guidelines that help properly diagnose and manage these eosinophilic disorders would also be useful.

Historically, chronic neutrophilic leukemia and atypical CML have been difficult to diagnose, but recently mutations in CSF3R have been reported in these groups and, depending on the specific type, could confer sensitivity to ruxolitinib or dasatinib. ${ }^{12}$ Myeloid (or lymphoid) neoplasms with eosinophilia and rearrangement of PDGFRA or PDGFRB, although not technically classified as MPNs, share similar clinical features and are exquisitely sensitive to imatinib; in contrast, FGFR1-rearranged neoplasms, also within this group, exhibit a poor prognosis, usually with rapid transformation to acute leukemia. This group also lacks guideline representation. Finally, recognition of MDS/MPN overlap syndromes (chronic myelomonocytic leukemia; juvenile myelomonocytic leukemia; MDS/MPN unclassifiable, including refractory anemia with ring sideroblasts and thrombocytosis and atypical CML) is increasing in clinical practice. Novel genetic alterations have been reported in patients with these disorders, including SETBP1 in $20 \%$ to $30 \%$ of patients with atypical CML. ${ }^{13}$ As novel diagnostic and therapeutic measures evolve in the overlap syndromes, hematologists and oncologists will need additional guidance in interpreting and applying findings to routine clinical practice.

Although expert consensus will play a role, the evidence base for decisionmaking is beginning to expand. BCR-ABL1-negative MPN and MDS/MPN overlap syndromes are coming of age, and the recognition of molecular genetic aberrations is changing the way hematologists and oncologists diagnose and treat these myeloid 
neoplasms. We believe the time is right for the creation of a comprehensive United States-based guideline that provides clear guidance on the approach to diagnosis, risk assessment, and management of patient populations with various forms of MPN. We are excited to be working with NCCN in the planning and development of these guidelines.

\section{References}

1. Stein BL, Gotlib J, Arcasoy M, et al. Historical views, conventional approaches, and evolving management strategies for myeloproliferative neoplasms. J Natl Compr Canc Netw 2015;13:424-434.

2. Bench AJ, White HE, Foroni L, et al. Molecular diagnosis of the myeloproliferative neoplasms: UK guidelines for the detection of JAK2 V617F and other relevant mutations. Br J Haematol 2013;160:25-34

3. Reilly JT, McMullin MF, Beer PA, et al. Guideline for the diagnosis and management of myelofibrosis. Br J Haematol 2012;158:453-471.

4. Harrison $\mathrm{CN}$, Bareford $\mathrm{D}$, Butt $\mathrm{N}$, et al. Guideline for investigation and management of adults and children presenting with a thrombocytosis. Br J Haematol 2010;149:352-375.

5. McMullin MF, Reilly JT, Campbell P, et al. Amendment to the guideline for diagnosis and investigation of polycythaemia/erythrocytosis. Br J Haematol 2007;138:821-822.

6. Tefferi A, Cervantes F, Mesa R, et al. Revised response criteria for myelofibrosis: International Working GroupMyeloproliferative Neoplasms Research and Treatment (IWG-MRT) and European LeukemiaNet (ELN) consensus report. Blood 2013;122:1395-1398.

7. Barosi G, Mesa R, Finazzi G, et al. Revised response criteria for polycythemia vera and essential thrombocythemia: an ELN and IWG-MRT consensus project. Blood 2013;121:4778-4781.

8. Barbui T, Barosi G, Birgegard G, et al. Philadelphia-negative classical myeloproliferative neoplasms: critical concepts and management recommendations from European LeukemiaNet. J Clin Oncol 2011;29:761-770.

9. Greenberg PL, Attar E, Bennett JM, et al. Myelodysplastic syndromes: clinical practice guidelines in oncology. J Natl Compr Canc Netw 2013;11:838-874.

10. O'Brien S, Radich JP, Abboud CN, et al. Chronic Myelogenous Leukemia, Version 1.2014. J Natl Compr Canc Netw 2013;11:1327-1340.

11. Akin C. Molecular diagnosis of mast cell disorders: a paper from the 2005 William Beaumont Hospital Symposium on Molecular Pathology. J Mol Diagn 2006;8:412-419.

12. Maxson JE, Gotlib J, Pollyea DA, et al. Oncogenic CSF3R mutations in chronic neutrophilic leukemia and atypical CML. N Engl J Med 2013;368:1781-1790.

13. Piazza R, Valletta S, Winkelmann N, et al. Recurrent SETBP1 mutations in atypical chronic myeloid leukemia. Nat Genet 2013;45:18-24. 